\title{
1 Effects of pore geometry on flowing foam dynamics in 3D printed porous media
}

2

$$
\text { Kofi Osei-Bonsu }{ }^{1} \text {, Paul Grassia }{ }^{2,3} \text { and Nima Shokri*1 }
$$

$3{ }^{1}$ School of Chemical Engineering and Analytical Science, University of Manchester,

4 Manchester, UK

$5^{2}$ Department of Chemical and Process Engineering, University of Strathclyde, Glasgow, UK 6

7

8

9

$10 *$ Corresponding author

11Dr. Nima Shokri

12School of Chemical Engineering and Analytical Science

13Room C26, The Mill

14The University of Manchester

15Sackville Street, Manchester, M13 9PL, UK

16Tel: 0441613063980

17Email: nima.shokri@manchester.ac.uk

18Group website: http://personalpages.manchester.ac.uk/staff/nima.shokri/ 


\section{Abstract}

21Foam flow in porous media is important in several environmental and industrial applications 22including soil remediation and enhanced oil recovery. The behaviour of foam is greatly 23influenced by transport properties of porous media, properties of foam and the fluid residing 24in porous media. We conducted a series of experiments to investigate the effects of pore 25geometry on foam flow in porous media and its implications for hydrocarbon displacement. 26We fabricated four porous media with well-defined pore throat size distributions, 27permeability and angularity by means of 3D printing technology. The models were initially 28saturated with oil. Gas and surfactant solution were subsequently injected into the model 29simultaneously for in-situ generation of foam to displace the oil. Displacement dynamics 30were recorded using an automatedie imaging setup. Analysis of the pore-scale images 31revealed that the injected pore volumes required for the initiation of foam generation 32decreased as the pore size of porous media increased presumably due to the lower entry 33capillary pressure. For the same pore throat size range, changes in the permeability due to 34increased number of pore throats did not appear to have a significant influence on the overall 35recovery of oil. Our results illustrate the impact of grain angularity on foam generation owing 36to its influence on the pore-to-throat aspect ratio and capillary pressure gradient.

\section{7}

38Keywords: Foam generation and propagation in porous media, Pore geometry and angularity, 39Minimum pressure gradient, 3D printing technology 


\section{Introduction}

44Contamination of soil by non-aqueous phase liquids (NAPLs) such as hydrocarbon-based

45products is a major environmental concern because of the potential danger it poses to the 46ecosystem (Hirasaki et al., 1996; Pennell et al., 1996). In many cases, contaminants migrate 47downwards and eventually reach the aquifer. During this process, portions of these 48contaminants are trapped in smaller pores due to capillary forces, serving as a long-term 49source of pollution to the ground water (Hirasaki et al., 1996). Furthermore, trapped oil 50phases can constitute a large fraction of oil initially in petroleum reservoirs. A conventional 51approach to displacing this trapped phase is to inject gas or surfactant solution into the 52reservoir (Payatakes, 1982; Lenormand et al., 1988; Pennell et al., 1996). These methods 53however suffer from several drawbacks such as gravity segregation and 54fingering/instabilities. To overcome these deficiencies, foam flooding has been proposed as a 55potential solution (Schramm and Wassmuth, 1994; Jeong et al., 2000). Foam is a dispersion of 56 gas in a liquid whereby the gas is separated by thin liquid films called lamellae. The liquid 57phase is generally made up of water and a foaming agent such as surfactant and/or 58nanoparticles to stabilize the generated lamellae and thus to improve the longevity of the 59bubbles (Schramm and Wassmuth, 1994; Binks and Horozov, 2005; Nguyen et al., 2014). In 60 contrast to the sole injection of gas or surfactant solution, foam is characterised by a higher 61apparent viscosity, which provides a favourable mobility ratio, suppressing the formation of 62fingers. Moreover, the higher apparent viscosity of foam in higher permeability layers can 63mitigate the undesired effects of the reservoir heterogeneity by diverting flow to low 64permeability regions (Hirasaki and Lawson, 1985; Grassia et al., 2014; Mas-Hernandez et al., 652015).

66Foam is generated in-situ by injection of alternating slugs of a surfactant solution and gas or 67by co-injection of surfactant and gas (Zeng et al., 2016; Osei-Bonsu et al., 2017a). Three 
68main foam generation mechanisms have been identified in the foam literature: snap-off, 69lamella division and leave behind (Ransohoff and Radke, 1988; Rossen and Gauglitz, 1990; 70Kovscek et al., 1994). Lamellae are created by snap-off mechanism in pore-throats when 71liquid gradually accumulates in gas-invaded pore throat and eventually bridges and blocks the 72throat (Kovscek et al., 1994; Gauglitz et al., 2002). Bubbles generated by this mechanism 73generally have sizes similar to the pore bodies. Snap-off is influenced by several factors 74including pore-to-throat aspect ratio, capillary number and pore geometry (Ransohoff and 75Radke, 1988; Kovscek et al., 1994). In lamella division, an existing bubble subdivides into 76two new bubbles upon stretching around branched points in porous media (Kovscek et al., 771994). Bubble creation by lamella division is also common when bubbles push against larger 78bubbles around a pore junction. The frequency of this foam generation mechanism may 79depend on several parameters such as the pore geometry, connectivity, and the initial size of 80bubbles (Kovscek et al., 1994). Foam generation by leave behind occurs when two gas 81menisci invade a liquid saturated pore, leaving behind a lens as the two menisci converge. A 82stable lens may form depending on the capillary pressure of the medium and the pressure 83gradient of the flow (Kovscek et al., 1994). Identifying the dominant foam generation 84mechanism is considered to be of importance as it can significantly influence the strength 85(apparent viscosity) of the foam. Foams generated by snap- off and lamella division have 86been observed to be stronger whereas leave behind produces weak foam (Kovscek et al., 871994).

88The performance of foam as a displacement fluid in porous media is controlled by numerous 89parameters ranging from the physiochemical properties of the foam (i.e. gas type/composition 90and surfactant) (Schramm and Novosad, 1990; Andrianov et al., 2012; Zeng et al., 2016), the 91oil properties (Schramm and Novosad, 1990; Nguyen et al., 2014; Osei-Bonsu et al., 2017a), 92to the properties of the porous medium (Schramm and Mannhardt, 1996; Kovscek and Bertin, 
932003; Ma et al., 2012; Gauteplass et al., 2015). The presence of oil is widely accepted to be 94one of the major deterrents to the performance of foam in porous media (Farajzadeh et al., 952012; Osei-Bonsu et al., 2017a). Oils with low viscosity and carbon chain lengths have been 96identified to be more detrimental to the stability of foam. Many experiments have revealed 97that different surfactants exhibit different tolerance to oil and hence potentially different 98effectiveness in porous media (Andrianov et al., 2012; Osei-Bonsu et al., 2017b).

\section{Foam stability in porous media is governed by the limiting capillary pressure $\left(P_{e}^{*}\right)$, above}

100 which foam coalescence becomes significant resulting in increasing the gas fractional flow

101(Farajzadeh et al., 2015). In other words, when water saturation drops below a limiting water

102 saturation ( $\left.S_{* *}^{*}\right)$, foam becomes excessively dry (and the lamellae too thin) to survive the 103imposed capillary pressure. The magnitude of the $P_{e}^{*}$-in particular depends on the 104permeability of the rock (Farajzadeh et al., 2015).

105The effects of porous media properties such as permeability on foam behaviour have been 106reported in numerous micromodel and core flooding studies in literature. It has been 107demonstrated that there is a minimum pressure gradient or critical injection velocity required 108for foam generation in porous media (Ransohoff and Radke, 1988; Rossen and Gauglitz, 1091990; Gauglitz et al., 2002). Gauglitz et al. (2002) showed that the minimum pressure 110gradient is an inverse function of permeability. In addition to influencing the pressure 111gradient required for foam generation, permeability affects the apparent viscosity of foam in 112porous media thus influencing its behaviour and performance as a displacing fluid (Osei113Bonsu et al., 2016).

114Although the effects of the presence of textural discontinuity such as permeability contrast on 115 foam flow in porous media have been investigated extensively in the past (Kovscek and 116Bertin, 2003; Ma et al., 2012), the influence of pore size and grain angularity on foam 
117generation and propagation in oil saturated porous media have received much less attention. 118Additionally, the majority of the previous work conducted on the effects of pore geometry has 119been done using packed sand or core samples in which the geometry and consistency are 120particularly challenging to control. With the aid of 3D printing technology, we have designed 121and fabricated porous media with well-defined characteristics in order to investigate the 122influence of the pore geometry on the dynamics of foam in oil-saturated porous media. The 1233D printing technique -allows for fast prototyping and fabrication of customized porous 124media with consistent and well controlled geometry which cannot be achieved with sandpack 125 or glassbead pack. This has enabled us to visualise and study the effects of pore throat size 126and grain angularity on foam behaviour and oil displacement by foam in porous media.

\section{Experimental Considerations}

\subsection{Design and fabrication of porous media}

129Prior to manufacturing the quasi-two-dimensional models used in this study, digital 130representations of the desired pore throat size distribution and the patterns were designed. 131'Rhinoceros’ CAD software package for 3D illustrations was used to create the pore 132networks. The pore network of each model was created from a 'Voronoi' diagram using a 133random array of points (Sahimi, 2011). Pore throat values were then randomly populated in 134the model. An algorithm was applied to either straighten or curve the edges of the grains in 135the model. Four different porous media with well-defined properties were fabricated and used 136in this study. Figure 1 shows an example of athe printed porous medium together with the 137cross sections of the printed porous media used in the present investigation. 


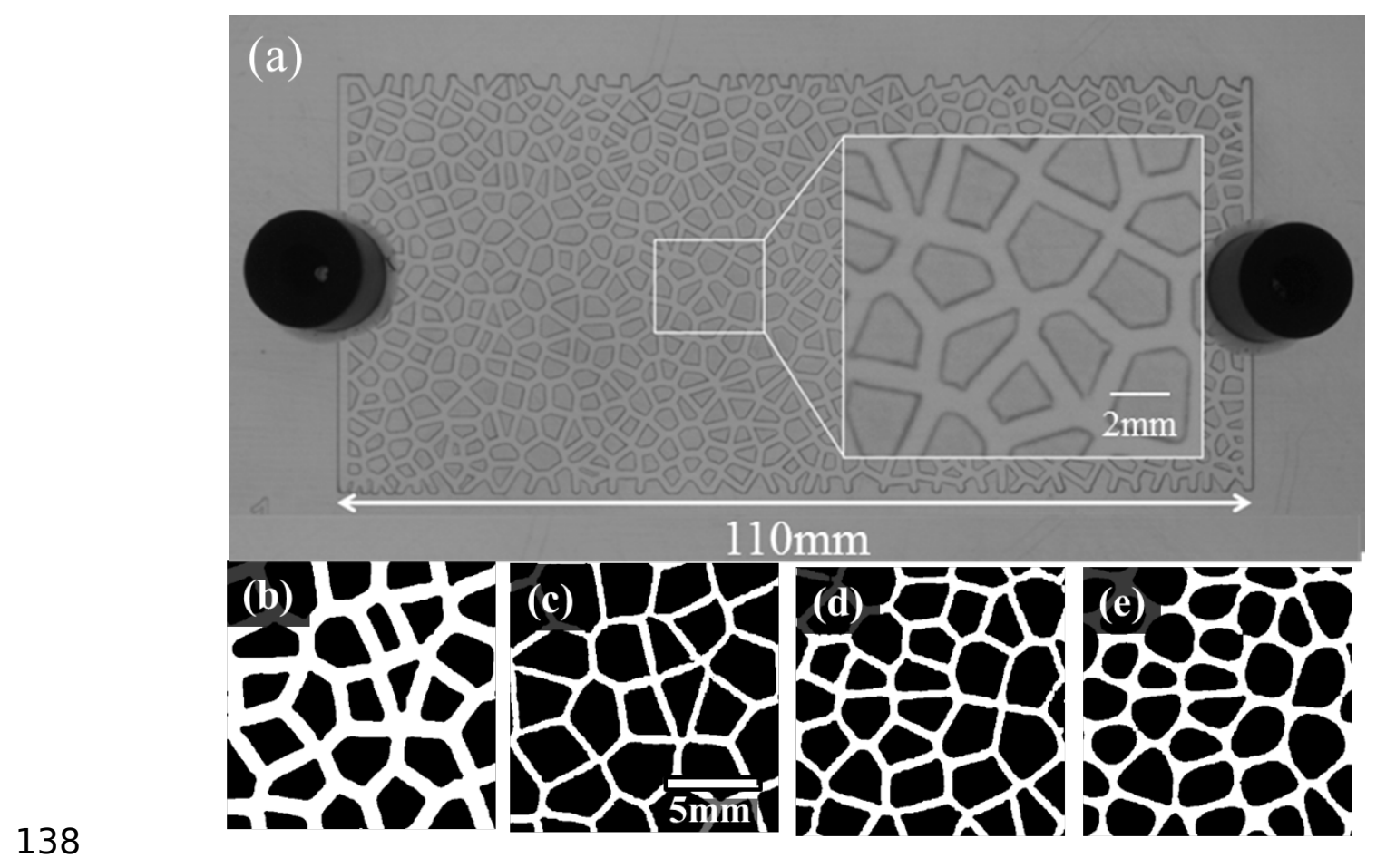

139Fig. 1 (a) Top view of one of the printed models with pore-throat-size distribution of 0.8-1.0 140mm with angular grains used in our study. (b), (c), (d) and (e) are cross-sections of the 141printed porous media with pore-throat-size distribution of $0.8-1.0 \mathrm{~mm}$ and porosity of $45.4 \%$ 142(angular grains; hereafter referred as Model A), $0.3-0.5 \mathrm{~mm}$ and porosity of $26.2 \%$ (angular 143grains; hereafter referred as Model B), 0.3-0.5 mm and porosity of 30.0\% (angular grains; 144hereafter referred as Model C) and 0.3-0.5mm and porosity of 36.0\% (round grains; hereafter 145referred as Model D), respectively.

146The properties of the porous media are provided in Table 1. The dimension of the models was $147110 \mathrm{~mm} \times 50 \mathrm{~mm}$ and the depth was half the maximum pore throat size in all four models. 148The spatial distribution of the grains for the models was the same and varied only in pore 149throat size and grain angularity respectively. The CAD models were converted to 150stereolithographic (STL) format which were then printed with an acrylic based resinmateriat 151(acrylic oligomer, Tritech, UK) by a high resolution Polyjet 3D printer (Objet 30 pro, 152Stratasys)(Osei-Bonsu 2017a and 2017b) The printer constructed the models from bottom to 
156washed out using a water jet cleaner after the printing was completed. The top of the printed 157models were sealed with a glass plate. Two holes of size $1 \mathrm{~mm}$ were perforated at the 158opposite ends of the top glass to allow injection of fluid into and out of the cell.

159Table 1. Properties of the printed porous media, number of Voronoi polygons refers to the 160number of grains in each model.

\begin{tabular}{|c|c|c|c|c|c|}
\hline $\begin{array}{c}\text { Model } \\
\text { (angularit } \\
\text { y) }\end{array}$ & $\begin{array}{c}\text { Pore throat } \\
\text { size } \\
\text { distribution }\end{array}$ & $\begin{array}{c}\text { Number } \\
\text { of Voronoi } \\
\text { polygons }\end{array}$ & $\frac{\text { Circularit }}{\mathrm{y}}$ & $\begin{array}{c}\text { Porosity } \\
(\%)\end{array}$ & $\begin{array}{c}\text { Permeabilit } \\
\text { y } \\
\text { (Darcy) }\end{array}$ \\
\hline $\begin{array}{c}\text { A } \\
\text { (angular) }\end{array}$ & $0.8-1.0 \mathrm{~mm}$ & 660 & $\underline{0.673}$ & 45.4 & 6.3 \\
\hline $\begin{array}{c}\mathrm{B} \\
\text { (angullar) }\end{array}$ & $0.3-0.5 \mathrm{~mm}$ & 660 & $\underline{0.644}$ & 26.2 & 4.7 \\
\hline $\begin{array}{c}\mathrm{C} \\
\text { (angullar) }\end{array}$ & $0.3-0.5 \mathrm{~mm}$ & 990 & $\underline{0.683}$ & 32.7 & 9.5 \\
\hline D (round) & $0.3-0.5 \mathrm{~mm}$ & 990 & $\underline{0.748}$ & 36.0 & 10.6 \\
\hline
\end{tabular}

161

\subsection{Fluid properties and experimental procedure}

163The foaming agent was made from a 1:1 blend of Sodium Dodecyl Sulphate (SDS) (Sigma, 164UK) and Cocamidopropyl betaine (The Soap Kitchen, UK) (2\% wt active content) with a 1650.25M NaCl (Sigma, UK) solution. This surfactant combination has been used in previous 166studies and has shown better tolerance to the presence of oil (i.e. foam films were more stable 167in the presence of oil) compared to the performance of the surfactants individually (Osei168Bonsu et al., 2015; 2016; 2017a). In this study, foam was generated in-situ by simultaneous 169injection of the surfactant solution and the gas into the porous medium via separate tubes. 170The surfactant stream was controlled by a syringe pump (Harvard Apparatus) and the gas by 
171a mass flow controller (Bronkhorst, UK). Nitrogen (98\% purity) was used as the gas phase. 172Two different types kinds of experiments were conducted in this study. In the first series of 173experiments, the models were fully saturated with water. Gas and surfactant solution were 174injected simultaneously to displace the water. In the second type of experimentapproach, the 175empty models were initially saturated fully with oil (Isopar V, $10.81 \times 10^{-3} \mathrm{~Pa}$ s- Brenntag, 176UK, see Table 2). Surfactant solution and gas were then injected simultaneously until all the 177oil in the models was displaced. A pressure transducer was used to record the pressure during 178the course of the experiment. The oil phase was stained by a red dye (oil red) to enhance the 179visual contrast with the displacing phase. The model was thoroughly cleaned with 180isopropanol and distilled water and dried before conducting the next experiment.

\section{Table 2. Oil properties}

\begin{tabular}{|c|c|c|c|c|}
\hline Oil & $\begin{array}{l}\text { Viscosity } \\
\left(\times 10^{-3} \mathrm{~Pa} \mathrm{~s}\right) \\
\end{array}$ & $\begin{array}{l}\text { Density } \\
\left(\mathrm{g} / \mathrm{cm}^{3}\right)\end{array}$ & $\begin{array}{l}\text { Surface Tension } \\
(\mathrm{mN} / \mathrm{m})\end{array}$ & $\begin{array}{l}\text { Interfacial tension (with } \\
\text { surfactant solution ( } \mathrm{mN} / \mathrm{m} \text { ) }\end{array}$ \\
\hline Isopar V & 10.81 & 0.815 & 25.44 & 0.130 \\
\hline
\end{tabular}

182

\subsection{Image analysis}

184Snapshots of the displacement process were captured using a high resolution monochromic 185camera (Teledyne DALSA Genie) mounted above the model. In order to improve the 186illumination and the quality of the captured images, a light box was placed beneath the 187porous media during the experiments. The output images had a resolution of $2560 \times 2048$ 188pixels with 8 bit grey levels. A code was developed in MATLAB to segment and distinguish 189between the oil, grains (solid phase) and foam (gas and surfactant solution). The segmented 190images were used to quantify the dynamics and efficiency of foam-oil displacement. The 191segmentation algorithms were similar to the ones described in Shokri et al. $(2008,2012)$ but 
192with minor alterations outlined as follows: the areas of the model saturated with oil and foam 193were distinguished by two main 'peaks' in the grey value histogram of each image. The first 194peak represented oil while the second peak corresponded to foam and the grains. A threshold 195was assigned to the point on the histogram between the peaks where the derivative of the grey 196value changed from negative to positive in order to distinguish the two peaks. The grains 197were then differentiated from foam using the image of the unsaturated model as a reference. 198The final image was presented in three grey values corresponding to the grains, foam and oil.

199

200

\section{Results and discussions}

202This section is laid out as follows. Section 3.1 considers the general influence of pore throat 203size on the flow properties in porous media. Then Section 3.1.1 and 3.1.2 deal with the 204dynamics of water and oil displacement by foam respectively. Section 3.2 considers the 205influence of grain angularity on foam behaviour. Finally section 3.3 discuss the displacement 206patterns of oil displacement by foam as influenced by pore geometry.

\subsection{Effects of pore throat size on foam flow in porous media}

208For porous media with the same connectivity and topology (e.g. Model A and B), increasing 209pore throat size (while maintaining the pore-throat aspect ratio, i.e. pore-size to throat size ) 210increases both porosity and permeability of the medium (see Table 1). Consequently, the 211pressure drop required for fluid displacement decreases according to Darcy’s law. At the pore 212scale, increasing pore throat size tends to decrease the entry capillary pressure (according to 213Young-Laplace equation; 
$214, P_{e}=2 \sigma / r$, where $P_{c}$ is the entry capillary pressure, $\sigma$ is the surface/interfacial tension and $r$ is 215the pore throat radius- and the pressure drop required for fluid mobilization in the throat

\section{6 fgiven by Poiseuille equation:}

$\Delta P=8 \mu l v / r^{2}$

(2)

$217 \Delta P=8 \mu l v / r^{2}$ where $\mu$ is the viscosity of fluid, $l$ is the length of the pore throat and $\mathrm{v}$ is the 218velocity). As a result of the strength of capillary forces, trapped phase saturation is expected 219to be higher for porous media with smaller pore throats. In the context of foam, pore throat 220size can have a crucial effect on the generation and stability of flowing foam in porous media 221due to its effect on capillary pressure. The following sections will discuss the observed 222influence of pore geometry on foam behaviour in porous media.

\subsubsection{Water displacement by foam}

224In all the experiments the gas and the surfactant solution were injected at a constant gas 225 fraction of $85 \%$ and a total volumetric flowrate of $11.76 \mathrm{ml} / \mathrm{h}$ (capillary number of $2.75 \times 10^{-6}$ 226and $5.50 \times 10^{-6}$ for Model A and B, respectively $(\mu / \sigma i$ where $\mu$ is the apparent

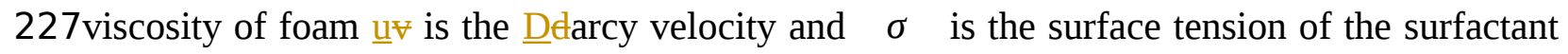
228solution). In order to fully understand the effects of the pore-throat size on foam generation in 229an oil-saturated model, experiments were first conducted with water as the residing fluid 230(which may-alse be considered relevant to foam flow in aquifers ). Foam generation and 231propagation waeres observed immediately after gas and surfactant solution were injected into 232the models. It was therefore expected that the imposed pressures created by the volumetric 233flow rate of gas and surfactant solution were above the minimum pressure gradient for foam 234generation in both models (Gauglitz et al., 2002). Additionally, water had a negligible 235influence on the stability of the foams hence little-minimal bubble coalescence was observed 236in both cases_(Osei - Bonsu et al 2017b). Figure 2a-f show the history of water displacement 237by foam for Model A and B. It can be observed that, although gas and surfactant solution 
238were injected as separate phases, stable foam generation occurred almost immediately 239resulting in a stable, piston - like displacement indicated by the red interface.

240Close inspection of the model revealed that the bubbles generated in the porous medium with 241the larger pore throat size (Model A) were more stable compared to Model B (smaller pore 242throat) due to higher rate of coalescence in Model B (higher capillary pressure). In addition, 243some clusters of water were trapped during displacement of water in spite of the higher 244injection capillary number in Model B. This is ascribed to the presence of continuous gas 245 flowing around such clusters during the early stages of water displacement in Model B due to 246relatively lower foam stability.

(a)

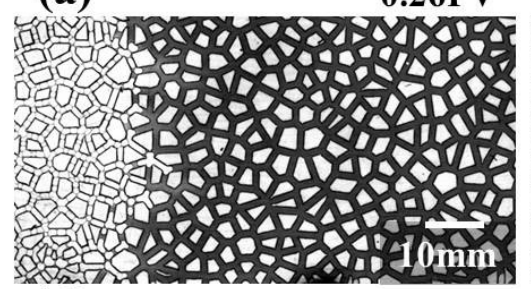

(d)

$0.25 \mathrm{PV}$

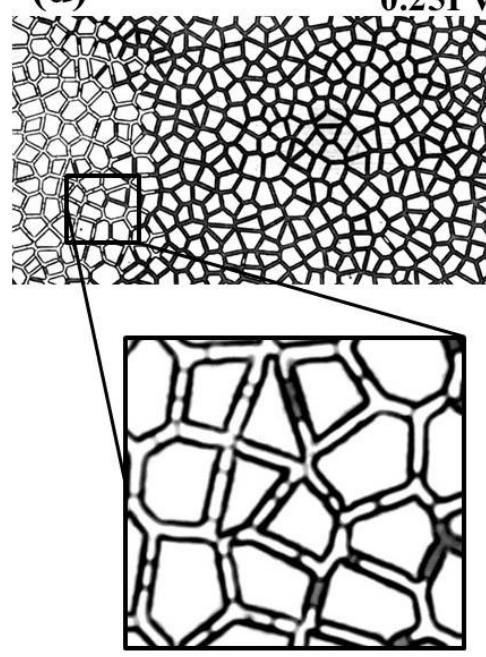

(b)

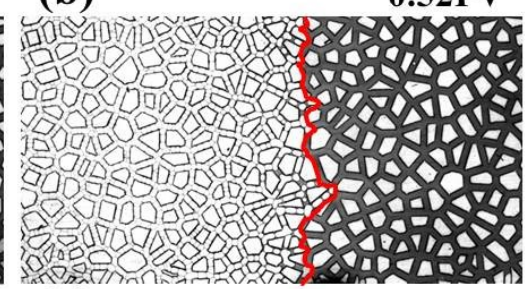

$0.52 P V$ (c)

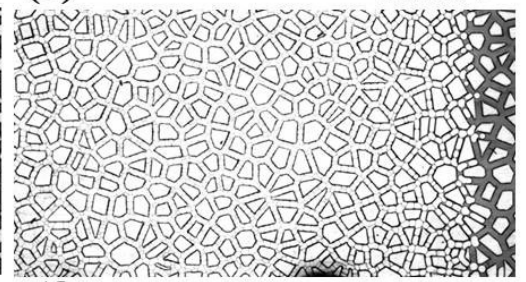

(f) 0.75PV
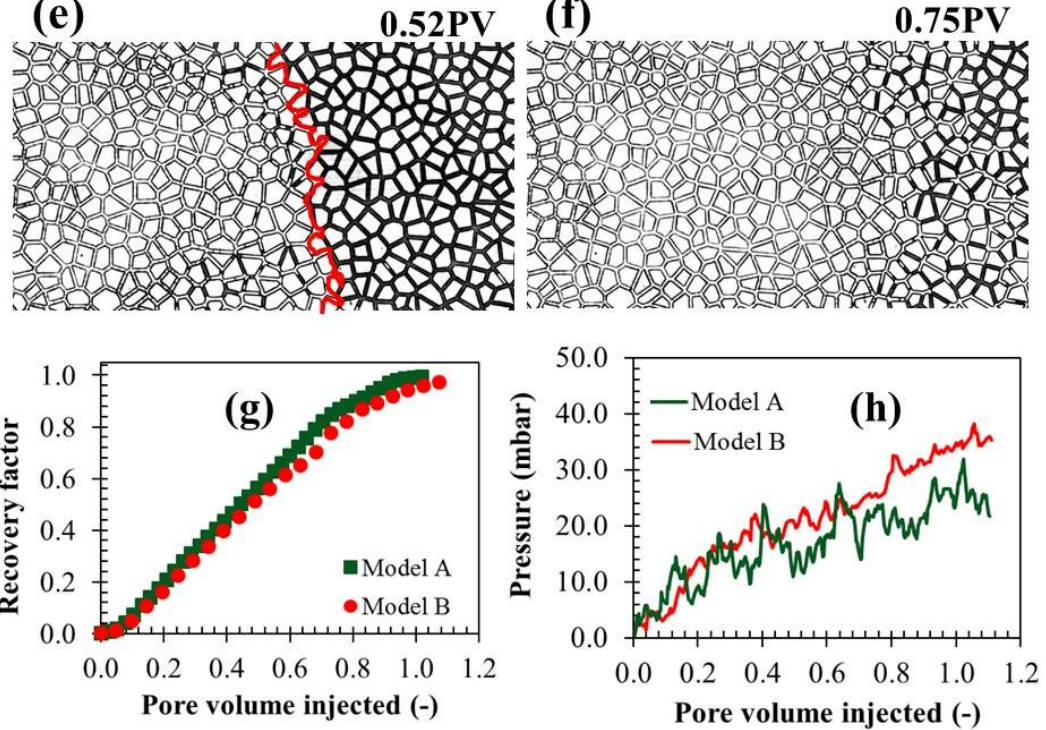

247

248Fig. 2. Water displacement by foam in porous media $(a-c)$ Model A and (d-f) Model B, The 249red curve represents the interface between foam and water. (g) Recovery of water as a 
250function of pore volumes of foam (gas and surfactant solution) injected (h) pressure drop 251across -Model A (green) and Model B (red) during displacement of water by foam.

252Irrespective of these effects -observed effects of the pore throat size on the dynamics and 253stability of foam, the water recovery factor, given by the amount of water recovered from the 254 model at that particular time divided by the initial amount of water in the model (i.e. full 255saturation), was not significantly influenced under these experimental conditions. The 256recovery of the residing phase in porous media depends on the mobility ratio between the 257displacing phase and the displaced phase (residing fluid) (Ma et al., 2012). Mobility is 258defined as the ratio between the relative phase permeability and the phase viscosity. 259Consequently, increasing the viscosity of the displacing phase decreases the mobility ratio 260leading to more favourable displacement efficiency. The apparent viscosity of foam can be 261computed from the steady state pressure drop using Darcy law-given by:

$$
\mu_{\text {app }}=\frac{k}{u} \frac{\Delta P}{L}
$$

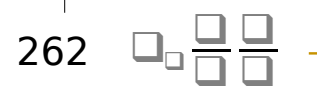

263where $\mu_{a p p}$ is the apparent viscosity of foam, $k$ is the permeability, $u$ is the 264superficiatDarcy velocity, $\Delta P$ is the pressure drop and $L$ the length of the model. The 265apparent viscosities of foam were $5.07 \times 10^{-3} \mathrm{~Pa} \mathrm{~s}$ and $2.21 \times 10^{-3} \mathrm{~Pa} \mathrm{~s}$ in both models $\mathrm{A}$ and 266B which are both larger than water, accounting for the stable displacement front indicated by 267the red curve in Fig 1 (b and e).

\subsubsection{Oil displacement by foam}

269Following the same procedure applied in the case of water displacement, oil displacement 270experiments were conducted, i.e., the same models were initially filled with the oil followed 
271 by the injection of foam. At the early stages of the injection, co-injection of the surfactant 272solution and gas resulted in no foam generation thereby allowing the surfactant solution and 273gas to flow as separate phases in the models even after breakthrough of the gas phase. This is 274mainly a consequence of the presence of oil in the porous medium which supressed the 275generation of foam (Osei-Bonsu et al., 2016, 2017a, 2017b). Foam generation in the porous 276 media began after a large fraction of oil had been displaced from the vicinity of the inlet of 277the model. During the initial phase of foam generation, some of the gas continued to 278propagate quickly through the porous medium as a separate phase while a fraction of the gas 279flowed as big bubbles (much larger than the diameter of pores). As fluid injection continued 280and foam generation progressed, the gas and surfactant solution no longer flowed as separate 281phases but as successive bubbles (discontinuous gas) due to increase in foam stability. Pore282scale analysis of the displacement process revealed that a smaller pore volume of gas and 283surfactant was required to initiate the generation of foam in Model A (average pore throat size 284of $0.9 \mathrm{~mm}$ ) in the presence of oil (see Figure 3) compared to Model B. According to Young285Laplace equation, the entry capillary pressure for gas to enter a pore throat in Model B is 286higher than Model A due to its smaller radius. However, in the previous section where the 287model was saturated with water, foam generation was not significantly influenced by the pore 288throat size. Analysis of Tthe entry capillary pressure may aid in understanding the reason for 289this observed phenomenon. Entry capillary pressure in this case was calculated using 290equation 1,- $\square_{\sqsupset} \square_{\square}$ where $\square_{\sqsupset}$ is the effective radius obtained from the expression for 291effective diameter, $d_{e}$ of a rectangular channel (Lenormand 1983);
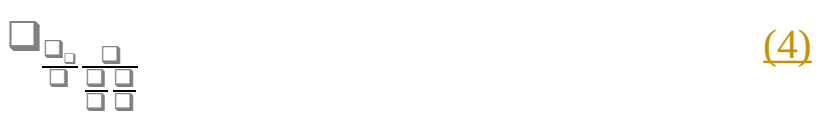

292

293where $\mathrm{F}$ e is a function approximately equal to 1.0 and $\mathrm{x}$ and $\mathrm{y}$ are the width and depth or the 294channel respectively. The entry capillary pressures for gas to invade water and oil filled 
296for gas to invade the porous media is higher for the water saturated model than for oil 297saturated model (for Model B it is $720 \underline{1055} \mathrm{~Pa}$ and $254 \underline{373} \mathrm{~Pa}$, respectively). However, for 298the case of water displacement the surfactant solution does not have to overcome any 299capillary pressure to invade a pore throat since water and the surfactant solution are miscible. 300As a result, there is no barrier for surfactant solution to enter pore throats where foam 301generation occurs.

302Contrariwise, capillarity becomes more significant to foam generation and stability in the 303case where the model is saturated with oil. In the scenarios considered here, the pressure 304required for surfactant solution to enterinvade a pore throat in Model B is at least twice that 305required for Model A_(1.90 Pa and 0.85 Pa respectively). In addition, the pressure drop 306required to mobilize oil from a smaller pore throat is higher than for the case of a larger one. 307The combined effect of these is the higher competition between surfactant solution and oil for 308pore throats (foam generation sites) as the pore throat size decreases. 

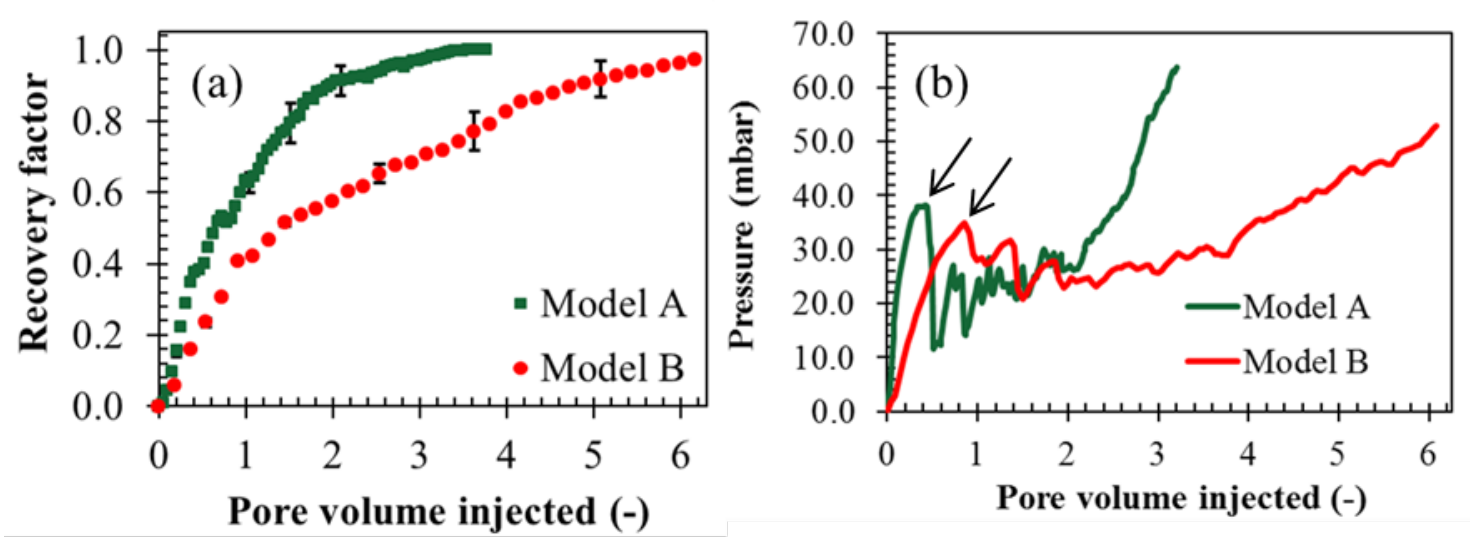

309
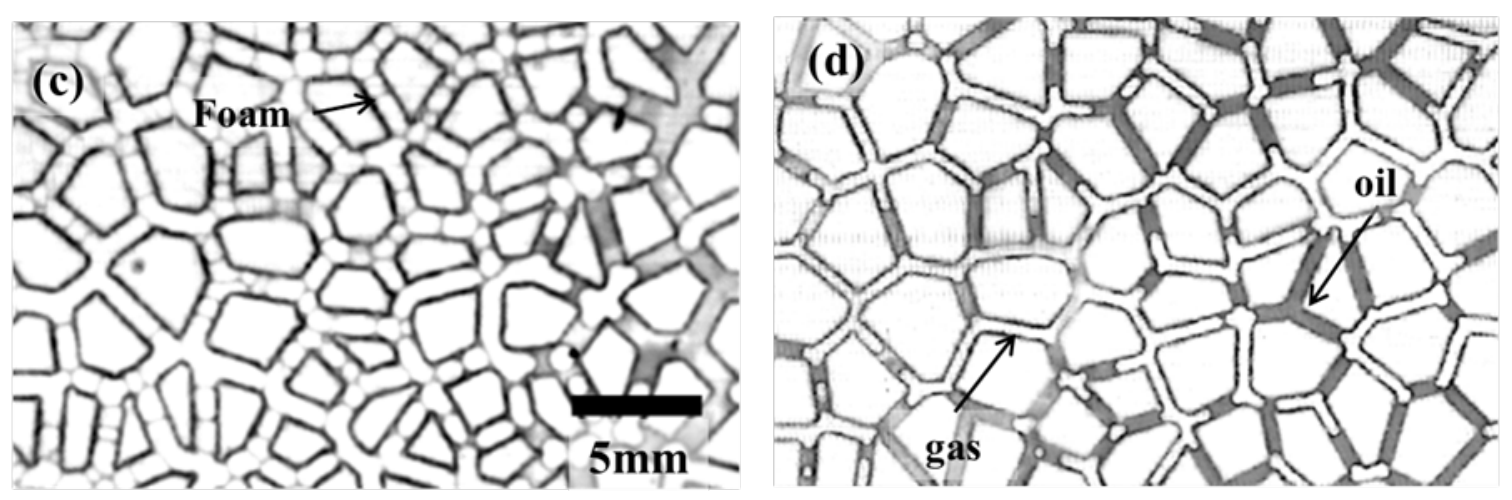

310Fig. 3. (a) Oil recovery efficiency by foam in porous media labelled as Model A (-average 311pore throat size of $0.9 \mathrm{~mm}$ ) and Model B (-average pore throat size of $0.4 \mathrm{~mm}$ ). Each 312experiment was conducted three times and the reported oil recovery curves indicate the 313average behaviour. (b) The pressure profile during oil displacement measured at the inlet of 314the model. Arrows represent breakthrough time. (c) and (d) Typical images of the porous 315media around the inlet after injection of $1 \mathrm{PV}$ of gas and surfactant solution into Model A and 316Model B, respectively.

317The segmented images were used to compute the oil recovery factor during the displacement 318process. Figure 3a shows the oil recovery for Model A and B as a function of total pore 319volume (PV) of the gas and surfactant solution injected. As observed from the figure, the oil 320recovery efficiency by foam reduces as the average pore throat size of the model decreases. 321In the case of Model A, almost all the oil was recovered after injection of 2 PV of foam (gas 322and surfactant solution) whereas in the case of Model B full oil recovery was achieved after 
323the injection of more than $6 \mathrm{PV}$. It is worth mentioning that the gas and surfactant solution 324(not foam) were responsible for oil recovery prior to foam generation as they flowed as 325separate phases in the models. This may account for the similar oil recovery rates during the 326injection of the initial 0.2 5PV. However, foam generation occurred much sooner in Model A 327compared to B accounting for the steady increase in oil recovery. As more stable lamellae are 328formed, the interaction between them increases. Additionally, gas bubbles are trapped in the 329 certain portions of the porous medium retarding the flow of foam and causing the pressure in 330the model to increase. The faster build up in pressure (gradient of curves in Figure 3b) after 331gas breakthrough (indicated by the black arrows) as the pore throat size increases ${ }_{\Perp}$ confirms 332the earlier and higher rate of foam generation and propagation of stable foam (Kovscek and 333Radke et al., 1994) in Model A. This is further evidenced by the snap-shots of the inlet region 334of the pore networks (Figure 3c and d). Figure 3d depicts no foam generation in Model B 335even after the injection of $1 \mathrm{PV}$ of gas and surfactant solution resulting in the dramatic 336decrease in the rate of oil recovery after the initial drive of oil by the gas phase. As a 337consequence of the delay in foam generation, a large fraction of the gas injected into the 338model followed the already established flow path resulting in little oil recovery. Additionally, 339trapping of oil during displacement contributes to more coalescence of foam in Model B 340(Figure 3d) decreasing the strength of foam, further decreasing the rate of oil recovery.

341

\subsection{Effects of grain angularity}

343In addition to the influence of the pore-throat size on the behaviour of foam in oil saturated 344porous media, the 3D printing technology enabled us to evaluate the effects of grain 345angularity on foam flow and generation. Model D was designed such that the positions of the 346grains were exactly the same as Model C with the exception that the grains were round as 
347opposed to irregular in the case of the latter. Figure 44 shows the oil displacement efficiency 348and the pressure profiles for the two models investigated under the same conditions. The oil 349recovery rate is identical for the two models from the onset of injection until about $1 \mathrm{PV}$ of 350gas and surfactant solution had been injected. This feature is attributed to the lack of foam 351generation within this time period. Consequently, as in the previous section, the oil was 352displaced by mainly gas and surfactant solution flowing through the model as separate 353phases. Under this displacement condition, the geometry had minimal impact on the recovery 354of oil. However, after this initial stage, the oil recovery rate increased noticeably in the case 355of Model D (round grains) compared to Model C (polygonal grains). This is ascribed to the 356lower entry capillary pressure of the round grains compared to porous media with angular 357grains becatse of the larger pore sizes which facilitated earlier foam generation leading to 358more efficient oil displacement(Nourozi Rad and Shokri 2014, Rabbani et al 2016) ${ }^{33-35}$.
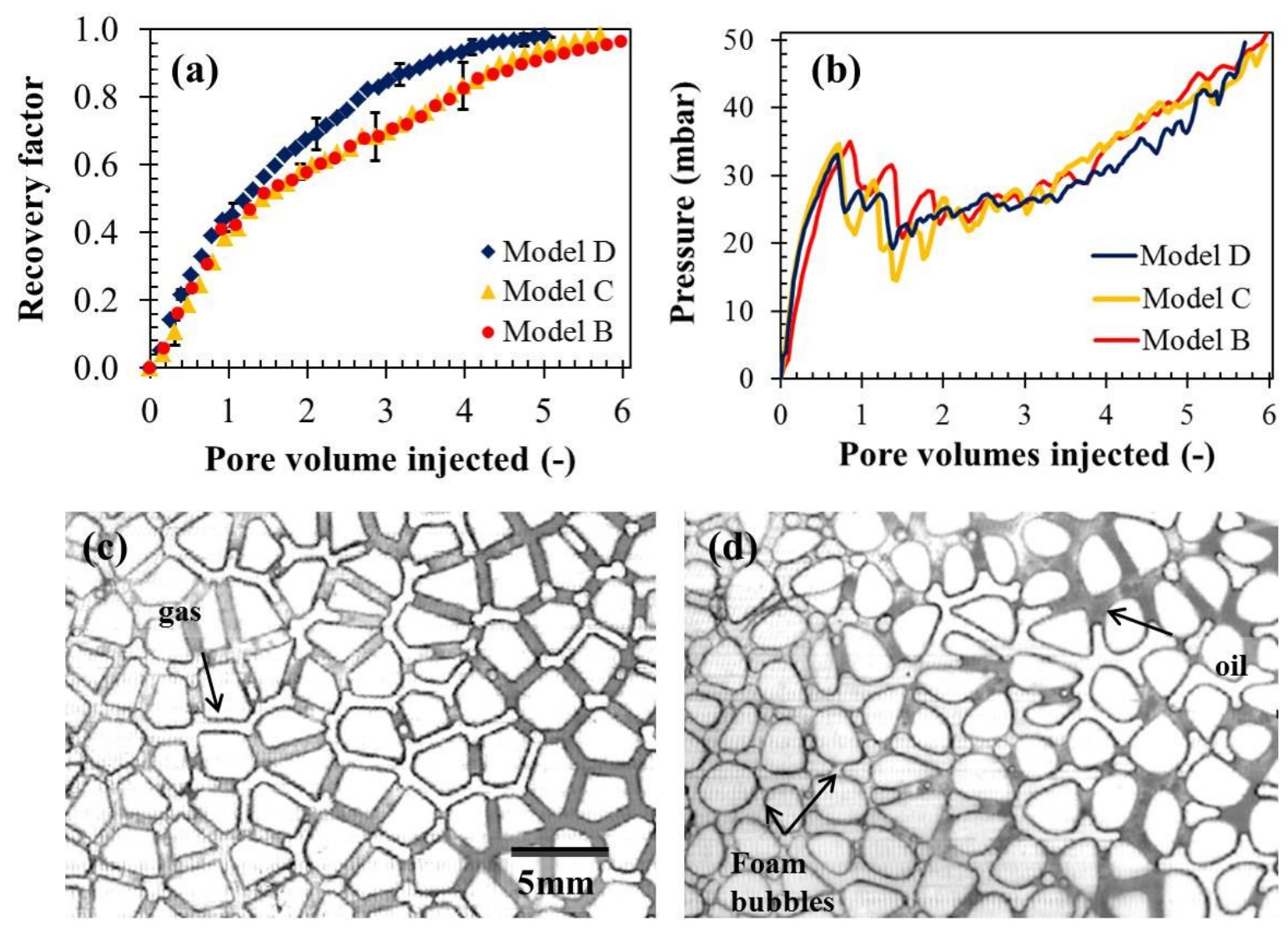
360Fig. 4. (a) Oil recovery as a function of pore volume injected in Model B, C (angular grains)

361 and Model D (round grains). (b) Pressure dynamics during oil displacement. (c) and (d)

362typical images showing the phase distribution around the inlet regions of model C and D

363respectively after 1.3 PV of surfactant and gas injected into the model. Foam generation in

364Model D was earlier than Model C.

365More importantly, the aspect ratio (ratio of pore to throat) plays a significant role in foam 366 generation by snap-off (Kovscek and Radke 1994, Roof 1970, Nguyen 2000). As shown in 367 the schematic in Figure 5, snap-off mechanism of bubble generation occurs when liquid 368surrounding the gas phase in a pore flows backward toward the throat as a result of capillary 369pressure gradient. This gradient is initiated by the differences in curvature and hence 370pressure between the gas phase in the throat $\left(\mathrm{P}_{\mathrm{at}}\right)$ and the pore $\left(\mathrm{P}_{\mathrm{ap}}\right)$ (subsequently leading to $\left.371 \underline{\mathrm{P}}_{\mathrm{wp}}>\mathrm{P}_{\mathrm{wt}}\right)$. The flow from the pore to the throat bridges the gas phase in the throat causing the 372 bubble to snap-off (Figure $5 \mathrm{~b}$ ). As the aspect ratio increases (thus as $\mathrm{R} / \mathrm{r}$ increases) the 373capillary pressure gradient increases, resulting in higher frequency of bubble generation. In 374the case of Model D, the roundness of the grains result in larger pores and hence higher 375aspect ratio (see Figure 5c). Consequently, this mechanism of foam generation is expected to 376be more dominant in the latter compared to Model C. 

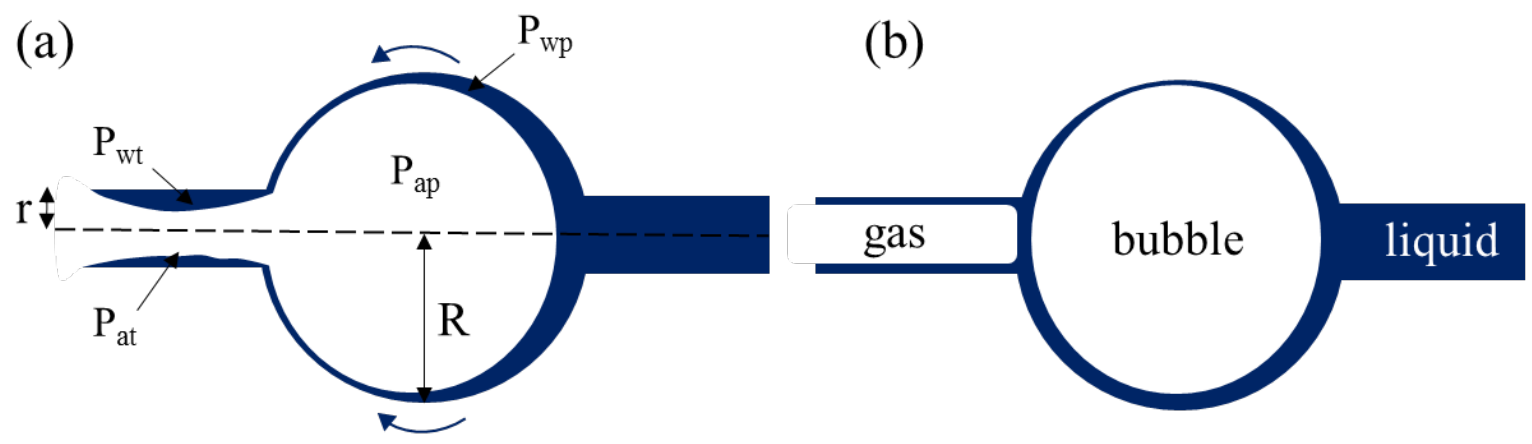

(c)

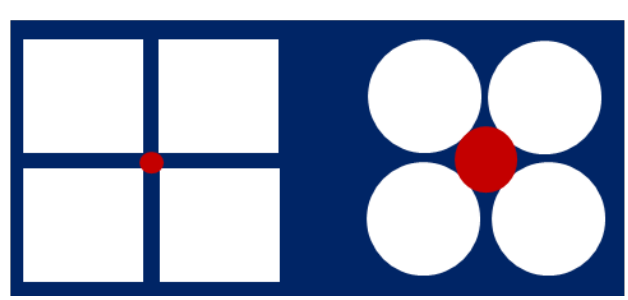

378Fig. 5 Schematic of snap-off mechanism during foam generation in porous media. (a) Liquid 379 flows from pore to throat to bridged gas phase due to capillary pressure gradient (b) 380Formation of a new bubble (c) pore geometry created by angular (left) and spherical grains 381 (right).

382 there is a higher pore-throat aspect ratio due to the bigger pore bodies in Model D which 383provides a higher capillary pressure gradient in the liquid phase (surfactant solution) between 384the pore body and pore throat (due to smaller eurvature in the pore body). This capillary 385pressure gradient drives surfactant solution from the pore into the pore throat to initiate 386bubble generation by the snap-off mechanism (Roof, 1970). 

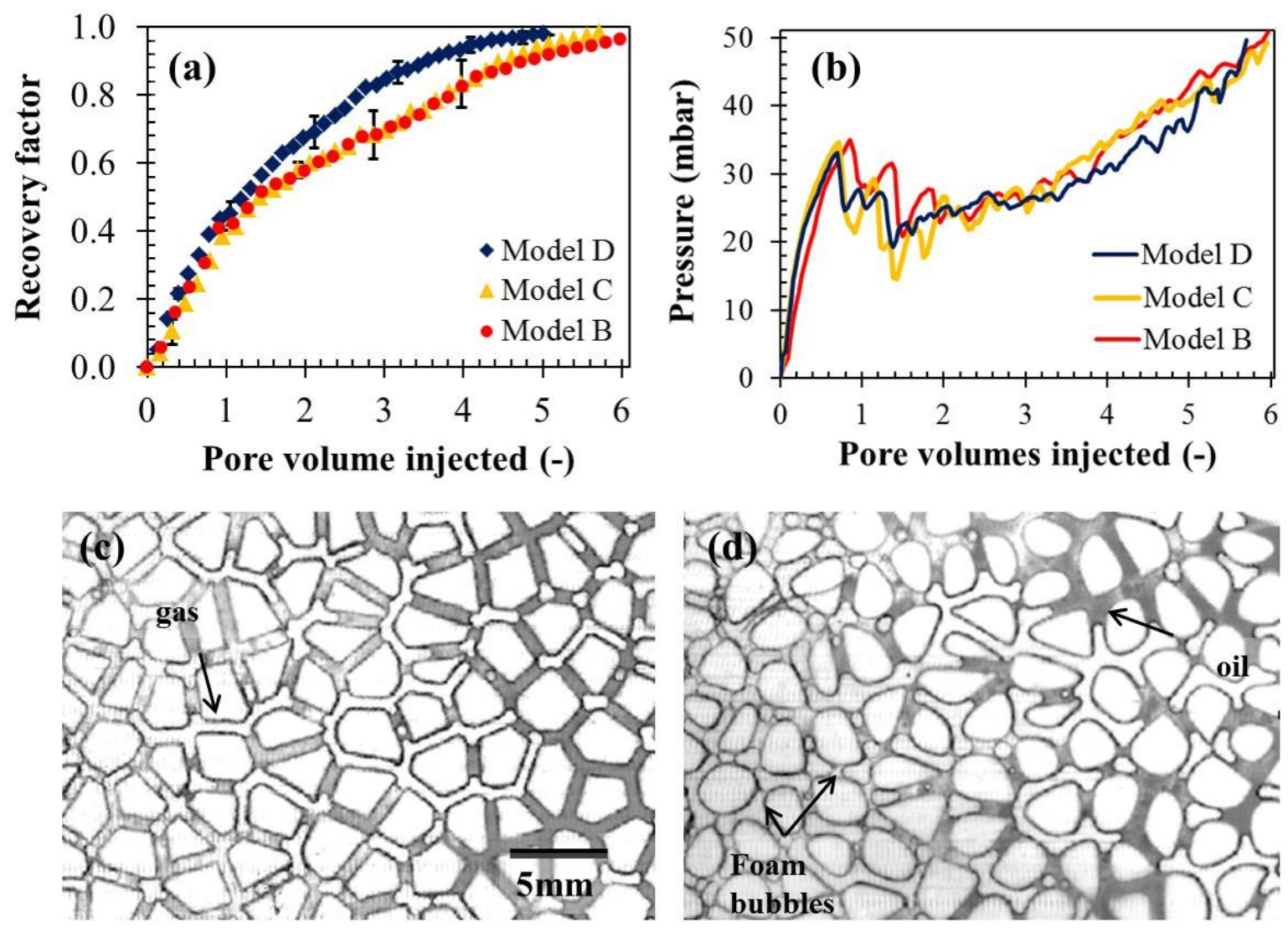

389Fig-4. (a) Oil recovery as a funetion of pore volume injected in Model B, C (angutlar grains)

390 and Model D (round grains). (b) Pressure dynamies during oil displacement. (c) and (d)-

391 typical images showing the phase distribution around the inlet regions of model $\mathrm{C}$ and $\mathrm{D}$

392 respectively after 1.3 PV of surfactant and gas injected into the model. Foam generation in-

393Model D was earlier than Model C.

394Unlike Figure 3b, where a glaring difference in the pressure profiles was observed, the 395variation in the pressure profiles for Model C and D was less due to the similar pore throat 396size. Nonetheless, careful inspection of Figure 44 b shows slightly higher pressure in Model D 397compared to C particularly (at least) during the initial phase of foam generation (between 3981.3PV and 2.5PV). This is indicative of more foam generation which is likewise confirmed 399by the close up shot of the inlet region depicted in Figure $44 \mathrm{c}$ and $\mathrm{d}$. 
400The effect of permeability on oil recovery was also investigated for porous media of the same 401pore throat size (Model B and C). In this case, permeability was modified by increasing the 402number of pores and throat while maintaining the dimensions of the pore throats. Model C 403consisted of 990 Voronoi polygons $(k=9.5 \mathrm{D})$ whereas Model B was made up of 660 404polygons $(\mathrm{k}=4.7 \mathrm{D})$. At the same injection capillary number $\left(5.50 \times 10^{-6}\right)$, oil recovery factor 405was not significantly affected by the permeability of the models potentially due to the low 406range of permeability investigated in this study. The corresponding pressure profiles were 407also similar in both cases suggesting that under these conditions, the pore throat size and 408angularity were more influential to the oil displacement by foam than the absolute 409permeability.

\subsection{Dynamics of oil displacement by foam}

411Figure $\underline{6} 5$ displays snapshots of the phase distributions after 0.5, 1.0 and $1.5 \mathrm{PV}$ of gas and 412surfactant solution injection in the three models used in our experiments. It can be observed 413 from the snapshots Figure 5 shows that the oil displacement process is influenced by the 414fingering phenomenon from the very onset of fluid injection into porous media. This is a 415consequence the lack of foam generation allowing the gas to penetrate the oil in the porous 416media. As fluid injection progressed, foam began to form in the porous media supressing the 417fingering effects. Figure $\underline{6} 5 \mathrm{c}$ shows the presence of a stable foam front in the case of Model A 418(the porous medium with the largest pores) after 1.5PV of injection. However, in the case of 419Model C and D (Figure $5 \mathrm{f}$ and i) fingering phenomena persisted even after $1.5 \mathrm{PV}$ due to the 420delay in the development of stable foam front. After nearly 2 PV of injection, stable foam 421fronts developed in the Models C and D (the images are not presented here). 


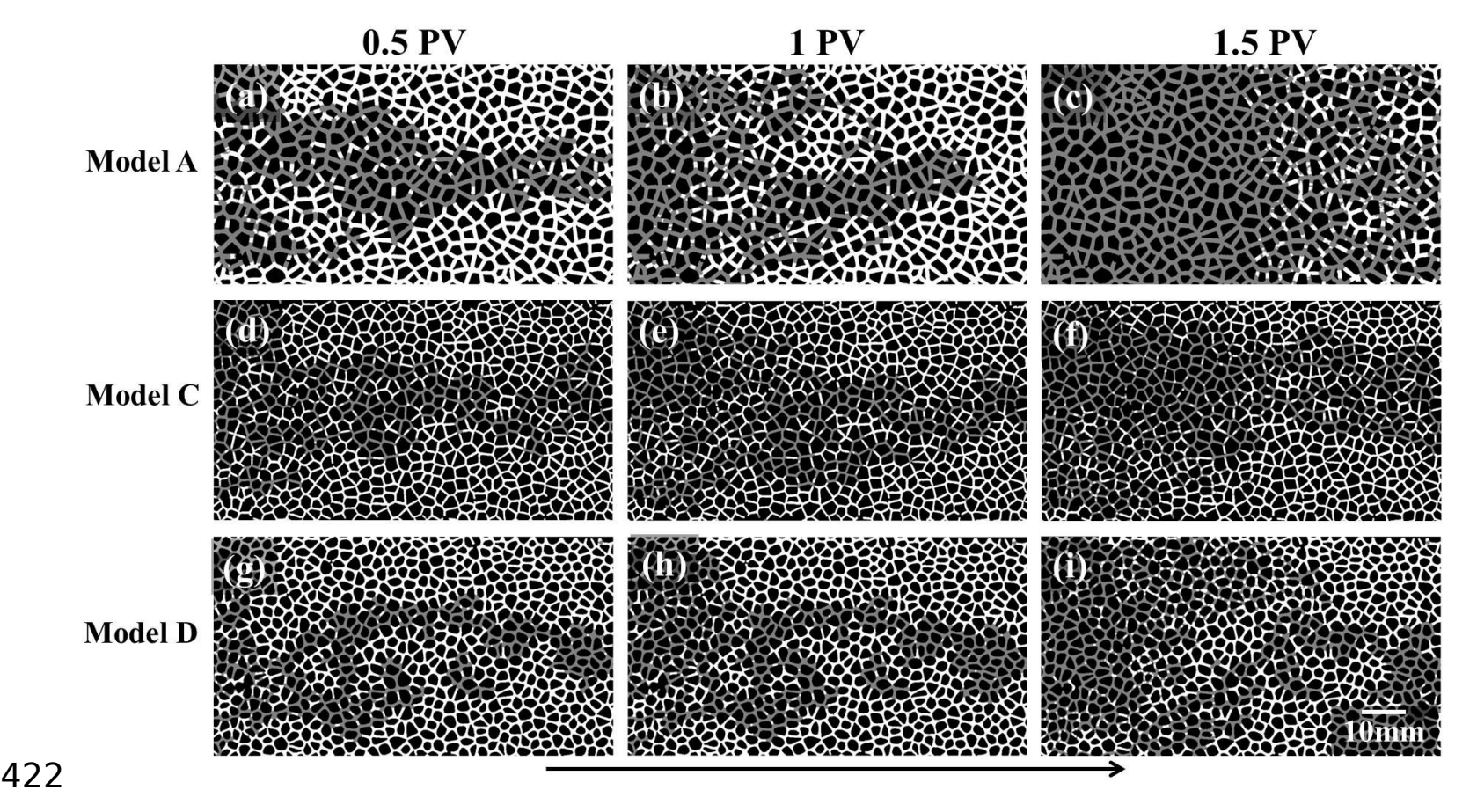

423Fig. 65. Dynamics and patterns of oil displacement by foam after the injection of 0.5, 1.0 and 4241.5 PV of gas and surfactant solution in Model A (a-c), Model C (d-f) and Model D (g -h). 425White, grey and black represent oil, foam (surfactant solution and gas) and solid grains 426respectively.

427The development and the evolution of the disconnected oil blobs formed during the 428displacement process can potentially be influenced by grain angularity and pore throat size as 429a result of the capillary pressure variation in porous media. Additionally, gas bubbles 430emerging at the foam front as a result of coalescence travelled rapidly through porous media 431amplifying the fragmentation of the oil phase. A typical example of this scenario is Figure 5c 432where disconnected oil blobs are observed ahead of the stable foam front.

433Analysis of the disconnected oil blobs as a function of pore volumes injected is presented in 434Figure 76 . The number of these oil fragments as a function of pore volume of fluid injected 435increased to a maximum (after about 1.2 PV for Model A and about 2.1 PV for Models B, C 436and D) but declined sharply as the remaining volume of oil in the model gradually decreased. 

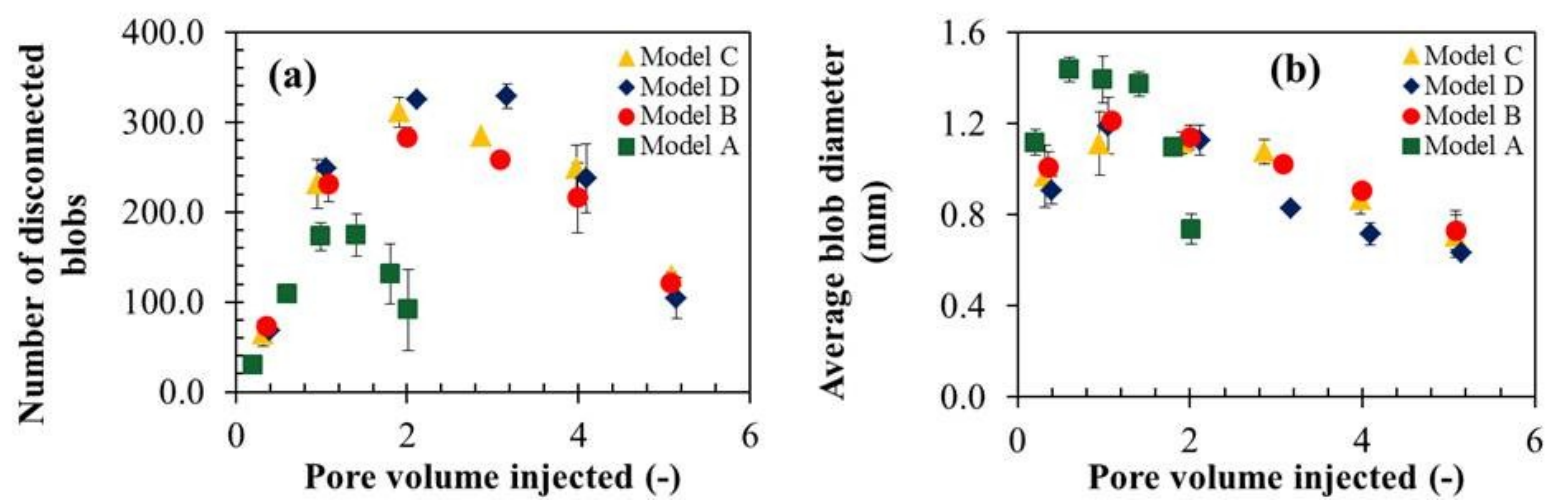

438Fig. $-\underline{76}$. The influence of the pore geometry on the statistical distribution of disconnected oil 439blobs during oil displacement by foam. The error bars represent the standard deviations.

440Figure 76 a shows that as the average pore throat size decreased, the number of isolated oil 441blobs due to fragmentation increased. The number of disconnected oil blobs were found to be 442larger in Model B, C and D compared to Model A. A possible reason for this observed result 443may be the higher capillary pressure associated with smaller pore throats in Model B, C and 444D compared to Model A. Additionally, the faster development of stable foam front and 445overall shorter oil recovery time in Model A compared to B and C also contributed to this 446observation. The average size of the oil blobs resulting from fragmentation was also found to 447be larger in the case of Model A owing to its larger pore throats (Figure 67b). Still within 448model A, the average size decreased sharply after about 1.8 PV of foam (surfactant and gas) 449had been injected as a large fraction of the oil in the model had been displaced at this point. 450In the case of Model B, C and D, neither the pore geometry nor the number of pores appeared 451to have a major influence on the fragmentation in the oil phase.

\section{Summary and Conclusions}

4533D printing technology enabled us to fabricate three customised porous networks with well454defined pore geometries. These were used to investigate the influence of average pore throat 455size and grain angularity on foam generation, propagation and the oil displacement efficiency 
456in porous media. Foam was generated in-situ following the co-injection of gas and surfactant 457solution into the porous media. Visualization of oil displacement by foam provided us with an 458opportunity to describe the effects of the pore geometry and angularity on foam generation 459and its relationship to the overall oil recovery efficiency. The following conclusions have 460been deduced from our investigation:

461 1. Pore geometry plays a crucial role in foam generation and propagation in oil saturated 462 porous media due to its influence on capillary pressure. For porous media with fixed 463 topology, increasing pore throat size decreases capillary pressure of the porous 464 medium which can improve foam generation and stability. In oil saturated models, the 465 influence of pore throat size on generation and propagation of foam is more 466 pronounced due to the additional negative effect of oil on the stability of foam 467 compared to water. Hence the number of pore volumes required for generation and 468 propagation of stable foam was smaller for porous media characterised by bigger pore throats.

470 2. For porous media with fixed pore throat size distribution, increase in permeability by

471 increasing number of pore throats (and pores) (Model B and C) did not result in any 472 noticeable effect on foam generation and hence oil recovery efficiency.

473 3. Foam generation is enhanced in porous media with round grains because of high pore-

474 to-throat aspect ratio which results in higher capillary pressure gradient between the 475 fluid at the pore throat and the pore body. This favours foam generation by snap-off 476 mechanism during the initial phase of foam generation.

477 Although flow in 2D micromodels has several limitations; the most notable being its inability 478 to capture the mixed wetting system encountered in natural occurring porous media, it is 479nonetheless able to reproduce adequately the underlying physics governing flow in porous 480 media making it a useful tool. With the development in 3D printing technology, we may be 481able to create porous media with channels of the same order as those occurring in real porous 
484 Appendix

485Table A1: The entry capillary pressure is expressed here is the pressure required for gas to 486invade a water/oil filled pore throat.

\begin{tabular}{|l|c|c|}
\hline \multicolumn{1}{|c|}{ Model } & $\underline{\text { Entry Capillary }}$ & $\underline{\text { Entry Capillary }}$ \\
& pressure (Pa) (water) & pressure (Pa) (oil) \\
\hline$\underline{\text { Model A }}$ & $\underline{474}$ & $\underline{165}$ \\
\hline$\underline{\text { Model B }}$ & $\underline{1055}$ & $\underline{373}$ \\
\hline
\end{tabular}

487

488These studies have been carried out in 2D porous media; more complex and intriguing 489 observations are expected in 3D system which offers an aventre for future investigation.

490

\section{Acknowledgements}

492We would like to acknowledge the UK Engineering and Physical Sciences Research Council 493(EPSRC) for providing the PhD studentship (EP/L504877/1) for Kofi Osei-Bonsu. Kofi 494would like to thank Harris Rabbani for the insightful discussions.

\section{References}

496Andrianov, A., Farajzadeh, R., Mahmoodi Nick, M., Talanana, M., Zitha, P.L.J.: Immiscible 497Foam for Enhancing Oil Recovery: Bulk and Porous Media Experiments. Ind. Eng. Chem. 498Res. 51, 2214-2226 (2012).

499Binks, B.P., Horozov, T.S.: Aqueous Foams Stabilized Solely by Silica Nanoparticles. 500Angewandte Chemie. 117, 3788-3791 (2005). 
501Farajzadeh, R., Andrianov, A., Krastev, R., Hirasaki, G.J., Rossen, W. R.: Foam-oil 502interaction in porous media: implications for foam assisted enhanced oil recovery. Adv.in 503Colloid Interf. Sci. 183-184, 1-13 (2012,).

504Farajzadeh, R., Lotfollahi, M., Eftekhari, A., Rossen, W., Hirasaki, G.: Effect of Permeability 505en Implicit-Texture Foam Model Parameters and the Limiting Gapillary Pressure. Energy \& 506Fuel. 29, 3011-3018 (2015).

507Gauteplass, J., Chaudhary, K., Kovscek, A.R., Fernø, M.A.: Pore-level Foam Generation and 508Flow for Mobility Control in Fractured Systems. Colloid and Surfaces A. 468, 184-192 509(2015).

510Gauglitz, A.P., Friedmann, F., Kam, I.S., Rossen, R.W.: Foam Generation in Homogeneous 511Porous Media. Chem. Eng. Sci. 57, 4037-4052 (2002).

512Grassia, P., Mas-Hernandez, E., Shokri, N., Cox, S.J., Mishuris, G., Rossen, W.R.: Analysis 513of a model for foam improved oil recovery. J. Fluid Mech. 751, 346-405 (2014).

514Hirasaki, G., Lawson, J.: Mechanisms of Foam Flow in Porous Media: Apparent Viscosity in 515Smooth Capillaries. SPE J. 25, 176-190 (1985).

516Hirasaki, G., Miller, C., Szafranski, R., Lawson, J., Akiya, N.: Surfactant/foam process for 517aquifer remediation. International Symposium on Oilfield Chemistry, Society of Petroleum 518Engineers, Surfactant/foam process for aquifer remediation (1996).

519Jeong, S.W., Corapcioglu, M.Y., Roosevelt, S.E.: Micromodel Study of Surfactant Foam 520Remediation of Residual Trichloroethylene. Environ. Sci. Tech. 34, 3456-3461 (2000).

521Kovscek, A., Bertin, H.: Foam Mobility in Heterogeneous Porous Media. Trans. Porous Med. 52252, 17-35 (2003).

523Kovscek, A.R., Radke, C.J.: Fundamentals of Foam Transport in Porous Media. Adv. Chem. 524242, 115-163 (1994). DOI: 10.1021/ba-1994-0242.ch003.

525Lenormand, R., Zarcone, C., and Sarr, A.: "Mechanisms of the Displacement of One Fluid by 526 Another in a Network of C.apillary Ducts," J. Fluid Mech. 135, 337-353 (1983) 
528Lenormand, R., Touboul, E., Zarcone, C.: Numerical models and experiments on immiscible 529displacements in porous media. J. Fluid Mech., 189, 165-187 (1988).

530Ma, K., Liontas, R., Conn, C.A., Hirasaki, G.J., Biswal, S.L.: Visualization of improved 531sweep with foam in heterogeneous porous media using microfluidics. Soft Matter. 8, 1066953210675 (2012).

533Mas-Hernandez, E., Grassia, P., Shokri, N.: Foam improved oil recovery: Foam front 534displacement in the presence of slumping. Colloid Surface A: Physicochem. Eng. Aspects. 535473, 123-132 (2015).

536Nguyen, P., Fadaei, H., Sinton, D.: Pore-Scale Assessment of Nanoparticle-Stabilized CO2 537Foam for Enhanced Oil Recovery. Energy \& Fuel. 28, 6221-6227 (2014).

538Norouzi Rad, M., Shokri, N.: Effects of grain angularity on $\mathrm{NaCl}$ precipitation in porous 539media during evaporation. Water Resour. Res. 50, 9020-9030 (2014).

540Osei-Bonsu, K., Shokri, N., Grassia., P.: Fundamental investigation of foam flow in a liquid541filled Hele-Shaw cell. J. Colloid Interface Sci. 462, 288-296 (2016).

542Osei-Bonsu, K., Grassia, P., Shokri, N.: Investigation of foam flow in a 3D printed porous 543medium in the presence of oil. J. Colloid Inter. Sci. 490, 850-858 (2017a).

544Osei-Bonsu, K., Grassia, P., Shokri, N.: Relationship between bulk foam stability, surfactant 545formulation and oil displacement efficiency in porous media. Fuel. 203, 403-410 (2017b).

546Osei-Bonsu, K., Shokri, N., Grassia., P.: Suffactant dependent fFoam stability in the presence 547and absence of hydrocarbons: From bubble- to bulk-scale. Colloid Surface A. 481, 514-526 548(2015).

549Payatakes, A.: Dynamics of Oil Ganglia During Immiscible Displacement in Water-Wet 550Porous Media. Ann. Rev. Fluid Mech. 14, 365-393 (1982).

551Pennell, K.D., Pope, G.A., Abriola, L.M.: Influence of Viscous and Buoyancy Forces on the 552Mobilization of Residual Tetrachloroethylene during Surfactant Flushing. Environ. Sci. 553Technol. 30, 1328-1335 (1996).

554Rabbani, H.S., Joekar-Niasar, V., Shokri, N.: Effects of intermediate wettability on entry 555capillary pressure in angular pores. J. Colloid Interf. Sci. 473, 34-43 (2016). 
556Ransohoff, T., Radke, C.: Mechanisms of Foam Generation in Glass-Bead Packs. SPE 557Reservoir Eng. 3, 573-585 (1988).

558Roof, J.: Snap-off of Oil Droplets in Water-Wet Pores. SPE J. 10, 85-90 (1970).

559Rossen, W.R., Gauglitz, P.A.: Percolation theory of creation and mobilization of foams in 560porous media. AIChE J. 36, 1176-1188 (1990).

561Sahimi, M.: Flow and Transport in Porous Media and Fractured Rock: From Classical 562Methods to Modern Approaches. 733 pp., Wiley- VCH Publishers, Weinheim, Germany 563(2011).

564Schramm, L.L., Mannhardt, K.: The effect of wettability on foam sensitivity to crude oil in 565porous media. J. Petrol. Sci. Eng. 15, 101-113 (1996).

566Schramm, L.L., Novosad, J.J.: Micro-visualization of foam interactions with a crude oil. 567Colloids and Surfaces. 46, 21-43 (1990).

568Schramm, L.L., Wassmuth, F.: Foams: Basic Principles. Adv. Chem. 242, 3-45 (1994). DOI: 56910.1021/ba-1994-0242.ch001.

570Shokri, N., Lehmann, P., Vontobel, P., Or, D.: Drying front and water content dynamics 571during evaporation from sand delineated by neutron radiography. Water Resour Res. 44, 572W06418 (2008).

573Shokri, N., Sahimi, M., Or, D.: Morphology, propagation dynamics and scaling 574characteristics of drying fronts in porous media. Geophys Res. Lett. 39, L09401 (2012).

575Zeng, Y., Ma, K., Farajzadeh, R., Puerto, M., Biswal, S.L., Hirasaki, G.J.: Effect of Surfactant 576Partitioning Between Gaseous Phase and Aqueous Phase on CO2CO2 Foam Transport for 577Enhanced Oil Recovery. Trans. Porous Med. 114(3), 777-793 (2016). 